\title{
Unambiguous Entropic Evaluation of a Complicated Construction Process
}

\author{
Eugene Barsky \\ Department of Industrial Engineering, Azrieli College of Engineering, Jerusalem, Israel \\ Email: eugene@jce.ac.il
}

Received 26 January 2016; accepted 28 March 2016; published 31 March 2016

Copyright (C) 2016 by author and Scientific Research Publishing Inc.

This work is licensed under the Creative Commons Attribution International License (CC BY). http://creativecommons.org/licenses/by/4.0/

c) (7) Open Access

\begin{abstract}
There exist very complicated creative fields of human activities. As a rule, these fields comprise a large number of internal objects involving huge amounts of people, where large financial and material resources are concentrated. Operative management of such objects is extremely difficult due to their complicacy and multidisciplinary character, as well as due to the absence of criteria allowing an unambiguous estimation of the completeness of works both at a separate object and at the created system on the whole. The availability of such a criterion will make it possible to redirect resources more rationally so that to save time and means while completing the intended project. The suggested criterion of such kind is based on the properties of entropy, which is the principal invariant of today's natural science. This parameter is perceived ambiguously, being permanently discussed in technical literature. Physical character of this parameter has been validated in detail by the Author [1] [2] who has shown its universality for the analysis of complicated systems at their modification. In the present paper, the development of such a criterion for a complicated engineering project is considered. However, this approach can be used for the analysis of complicated technical projects in other fields of human activities, as well.
\end{abstract}

\section{Keywords}

Entropy, Evaluation, Transport, Optimization, Probability, Operation Research, Criterion

\section{Introduction}

Modern construction objects often represent complicated multidisciplinary and multilevel projects. By way of example, we can consider the construction of a railway segment, which involves such main activities as excavation works, rails and crossties laying, erection of intermediate station premises, development of a branched systems of electric power supply, signalization and blocking and, finally, neighborhood improvement. As a rule, 
there is a manager at each sector of such construction. The amount of such managers can be large enough, and all of them can have different qualification and experience. As a rule, any construction is directed by a single person who is entrusted with the financing of the entire project. He is responsible for the progress of site works and the time frame of their execution. It is very important for him to estimate correctly the progress of works at any moment, to reveal backward sectors, to coordinate all the activities. It is important also for the managers of smaller subdivisions, for example, those responsible for the buildings of the station premises with a complete set of internal equipment, or for those responsible for rails laying, bridge building, etc. Sometimes subordinate managers are unable to evaluate objectively the completeness of works entrusted to them. Besides, even if subordinate managers can give close-to-reality estimations on the basis of their own experience and intuition, it is rather difficult for a chief manager to make an unambiguous conclusion about the general situation at the construction site on the basis of the obtained information, the more so because this situation changes daily.

It appears possible to develop a methodology that can provide a single-valued estimation of the status of both the entire project and its constituents. The availability of such a methodology will make it possible to simplify the building management and avoid mistakes and distortions in the course of it, which will save time and decrease construction costs. To develop such a methodology, it is necessary to solve the problem of numerical evaluation of the status of complicated systems.

\section{Unambiguous Evaluation of Complicated Systems}

The main problem is reduced to the development of methodology of unambiguous numerical estimation of the state of a system of any complicacy. As a rule, complicated systems consist of several components, their number being different. It is very important to have a notion about the relationships between these components in the system. Rather often, if the number of components is small, it is sufficient to determine the ratio between them. But the most complete idea is provided by the usual percentage reduced to $100 \%$. The estimation can be also reduced to fractions of unity, which correlates it with probability. The probability is determined as

$$
P\left(x_{i}\right)=\frac{x_{i}}{\sum x_{i}}
$$

Here $x_{i}$ has any dimension (tons, dollars, kilograms, percentage, pieces, etc.)

If a system consists of two components, a specified content of one component automatically defines the content of another, since the sum of their contents is unity. Hence, for a binary system, a single-valued estimation can be obtained specifying the content of one of the components.

The situation is different if a system consists of more than two components. In this case, the content of one component does not define those of others. If the contents of all components of the system are specified simultaneously, it gives a multiple (and not a single-valued) estimation. Therefore, in this case we use other characteristic instead of the probability - a measure of uncertainty introduced by Hartly in 1929 [3] and then used by Shannon in 1948 when developing the theory of information. The notion of the measure of uncertainty can be clarified by the following elementary example. We assume that a random value $x_{i}$ has $k$ equiprobable outcomes. (When tossing a coin, $k=2$, when casting a die, $k=6$ ).

According to the probability definition,

$$
P\left(x_{i}\right)=\frac{1}{k}
$$

The uncertainty is a function of the number of outcomes, and it can be denoted by $f(k)$. This function must satisfy the following requirements:

1) The more complicated is the system composition (i.e., the greater the number of outcomes), the higher must be the measure of uncertainty, i.e. $f(k)$ must be a monotonically increasing function.

2) If a random quantity has one outcome, i.e. the system consists of elements of the same type, the uncertainty cannot exist, i.e.

$$
f(1)=0
$$

3) If there are two independent systems, one of them having $k$ outputs, and another $l$ outputs, the total number of outputs must be $f(k l)$. However, this function should not contain a product of uncertainties, since 
the single-output character of one system eliminated the uncertainty of the combined system, which is false. Hence, the uncertainty of a combined system must possess the additivity property:

$$
f(k l)=f(k)+f(l)
$$

Shannon [3] has shown that the only function of the number of outputs satisfying all these requirements is a quantity proportional to the logarithm of the number of outputs.

$$
f(k)=A \log k=H(x)
$$

where $A$ is the proportionality coefficient; $H(x)$-the uncertainty of a random value; $\log k$-a quantity determined with the accuracy up to a constant, because the base of logarithm is not determined yet.

The dependence (4) can be interpreted as the static entropy, which determines, together with the dynamic entropy, the uncertainty of any transformation process in nature and technology. A detailed substantiation of this issue would take too much space in the article, and those interested can refer to the Author's book [1].

\section{Entropy of a Binary System}

A binary system consists of two components. The simplest example of such a system is a bulk mixture of a grinded material consisting of fine and coarse particles. The boundary between these materials is usually specified by the mesh size of a certain sieve. Particles passing through it are considered fine, and those remaining on the sieve-coarse. Imagine a system having $N_{1}$ coarse particles and $N_{2}$ fine ones, their sun being

$$
N_{1}+N_{2}=G
$$

According to (1), the content or probability of coarse particles in a system is

$$
P_{1}=\frac{N_{1}}{G}
$$

and the probability of fine particles is

$$
N_{2}=\frac{N_{2}}{G}
$$

According to Fermi's statistics, the number of outcomes (ways of alternation) for such a system is

$$
k=\frac{G !}{N_{1} ! N_{2} !}
$$

According to (4), this can be expressed by a relation

$$
H(x)=A \log k=A\left(\log G !-\log N_{1} !-\log N_{2} !\right)
$$

If the quantities $G ; N_{1}$ and $N$ are large (exceeding 1000), the logarithms of factorials can be computed exactly enough using Stirling's formula

$$
\log Q ! \approx Q(\log Q-1)
$$

Taking this expression into account, Equation (7) can be written as

$$
H(x)=A\left[G(\log G-1)-N_{1}\left(\log N_{1}-1\right)-N_{2}\left(\log N_{2}-1\right)\right]
$$

Removing brackets, we finally obtain

$$
H(x)=-A G\left[\frac{N_{1}}{G} \log \frac{N_{1}}{G}+\frac{N_{2}}{G} \log \frac{N_{2}}{G}\right]
$$

Taking (5) and (6) into account, this expression can be written as

$$
H(x)=-A G\left(P_{1} \log P_{1}+P_{2} \log P_{2}\right)
$$




\section{Entropy of Multicomponent Systems}

We will show that a dependence of (8) type is valid for multicomponent systems, too. Imagine that a system of $G$ particles of crushed material consists of $n$ size classed determined by a set of $(n-1)$ sieves.

We denote their content in each class by $N_{1} ; N_{2} ; N_{3} ; \cdots ; N_{i} ; \cdots ; N_{n}$. It is clear that $\sum_{n} N_{i}=G$.

Imagine that these particles are obtained from polymetallic ore, and they also differ by density within each size class. It is convenient to express the contents of such a composition by a matrix

$$
\left|\begin{array}{ccccccc}
M_{11} & M_{12} & M_{13} & \ldots & M_{1 i} & \ldots & M_{1 n} \\
M_{21} & M_{22} & M_{23} & \ldots & M_{2 i} & \ldots & M_{2 n} \\
\vdots & & & & & & \\
M_{j 1} & M_{j 1} & M_{j 3} & \ldots & M_{j i} & \ldots & M_{j n} \\
\vdots & & & & & & \\
M_{m 1} & M_{m 2} & M_{m 3} & \ldots & M_{m i} & \ldots & M_{m n}
\end{array}\right|
$$

where $M_{j i}$ is the content of particles with the density $j$ in size class $i$.

Clearly,

$$
\sum_{i}^{n} \sum_{j}^{m} M_{j i}=G
$$

By definition, the probability of $j i$-th particles contents in the system is

$$
P_{j i}=\frac{M_{j i}}{G}
$$

It follows that

$$
\sum_{i}^{n} \sum_{j}^{m} P_{j i}=1
$$

The total number of outcomes for the mentioned mixture of particles is

$$
k=\frac{G !}{\prod_{i=1}^{n} \prod_{j=1}^{m} M_{j i}}
$$

Hence, the mixture composition entropy in the system is expressed by the dependence

$$
H(x)=A \log k=A\left[G(\log G-1)-\sum_{i=1}^{n} \sum_{j=1}^{m} M_{j i}\left(\log M_{j i}-1\right)\right]
$$

Taking into account the relation (9), we obtain

$$
H(x)=-A G \sum_{i=1}^{n} \sum_{j=1}^{m} P_{j i} \log P_{j i}
$$

Since the probability is expressed in fractions of unity in the relations (8) and (10), $G$ can be equated to unity, and the components content can be expressed by their probability. The coefficient $A$ can take any value except zero and infinity. For the sake of computations convenience, we also assume that $A=1$. Then an expression for the evaluation of entropy of the mixture can be written as

$$
H(x)=\sum_{i=1}^{n} \sum_{j=1}^{m} P_{j i} \log P_{j i}
$$

As for the bases of logarithms, they can be whatever, any assumed values give results differing by a constant. At the comparison of successive computations in the process of the system change, the influence of the loga- 
rithm base is leveled. Therefore, for greater convenience in practical computations, we can recommend to use decimal logarithms, and for theoretical derivations-natural logarithms. There are no distinctions in kind between them.

Having clarified all these nuances, we can pass to the consideration of the general situation at the analysis of the status of a complicated building project in the process of its erection.

\section{Evaluation of Completeness of a Complicated Object in the Process of Construction}

It is known [4] that the construction of industrial enterprises is characterized by a diversity of erected objects and their versatility involving various kinds of works. It is usually very difficult to evaluate the extent of completeness of separate elements at the stage of the construction of such objects. And even if one manages to do this as impartially as possible, it is hard to make a conclusion about the state of the entire object at some moment of time on the basis of multiple evaluations. This problem can be solved using the parameter of static entropy. It is best of all to demonstrate this on a definite object.

For this purpose, we consider the construction of a railway segment in general. Imagine that according to the project, it is planned to build several stations at this railway equipped with railway terminals of various levels depending on the population size in the particular locality and on some other causes. At some stations it is planned to build repair workshops of various levels and even several depots, other stations being without all this. Some sections of the railroad bed will be trailed in a plain terrain, others in hilly and mountainous terrain. It means that besides excavation works for the railway track along the entire railroad, it becomes necessary to build bridges and tunnels. All the objects can be totally different.

A schematic diagram of such project is shown in Figure 1. In this diagram, railway terminals are enumerated as $x_{1} ; x_{2} ; x_{3} ; \cdots ; x_{i} ; \cdots ; x_{n}$. Road segments between them are enumerated according to the number of terminal located to the left and denoted by $y_{1} ; y_{2} ; y_{3} ; \cdots ; y_{i} ; \cdots ; y_{n}$.

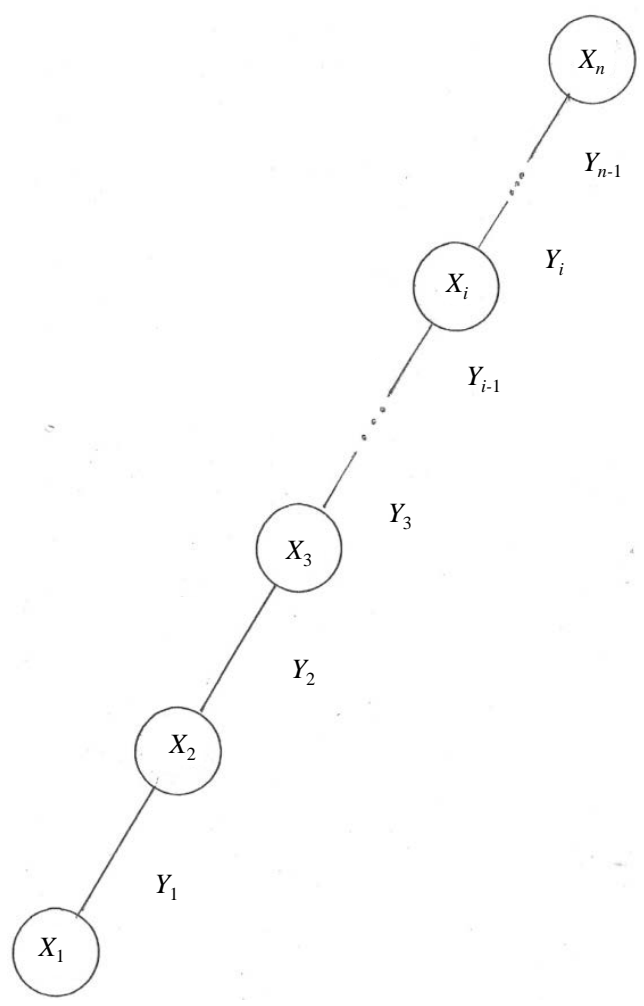

Figure 1. Respectively, we denote a list of works at the station by the symbol $x$ and the road sections by $y$. 
Approximate incomplete list of works at the stations:

$x_{1}$ - excavation works;

$x_{2}$-building foundation;

$x_{3}$-walls and roof;

$x_{4}$-plasterwork;

$x_{5}$-plumbing;

$x_{6}$-electrical work;

$x_{7}$-communication means;

$x_{8}$ - automation;

$x_{9}$-workshops,

etc., up to:

$x_{m}$-arrangement of green spaces.

For the railroad track:

$y_{1}$-on-the-site planning;

$y_{2}$-leveling and earthing;

$y_{3}$-bridges;

$y_{4}$-overhead roads;

$y_{5}$-laying crossties;

$y_{6}$-laying rails;

$y_{7}$-building of railway points and traffic lights;

$y_{8}$-automation and block systems;

$y_{9}$-communication lines, electricity,

etc, up to:

$y_{s}$-land improvement, clearing of rubble and debris.

Naturally, the presented list of works is incomplete, otherwise it would take up the volume of a whole book. We just consider the principle of unambiguous evaluation of works in such a complicated case.

Before starting the construction, each kind of works is estimated, e.g., in dollars. In a general form, such expenses for the construction of the first station can be written as follows:

$$
Q\left(x_{11}\right)+Q\left(x_{12}\right)+Q\left(x_{13}\right)+\cdots+Q\left(x_{1 i}\right)+\cdots+Q\left(x_{1 m}\right)=Q_{1}\left(x_{1}\right)
$$

Dividing the left side by the right one, we obtain the share of expenses for each kind of works at the first station, which is written as

$$
P\left(x_{11}\right)+P\left(x_{12}\right)+P\left(x_{13}\right)+\cdots+P\left(x_{1 i}\right)+\cdots+P\left(x_{1 m}\right)=1
$$

Each of these summands can be interpreted as a characteristic of the relative cost of works. For each of these works at the construction of the first object, the static entropy can be determined using the following formula:

$$
H\left(x_{1, i}\right)=P\left(x_{1 i}\right) \ln P\left(x_{1 i}\right)
$$

As a result, we can write for the entire object

$$
H\left(x_{11}\right)+H\left(x_{12}\right)+H\left(x_{13}\right)+\cdots+H\left(x_{1 i}\right)+\cdots+H\left(x_{1 m}\right)=H\left(x_{1}\right)
$$

The total value $H\left(x_{1}\right)$ determines the complicacy of building the station denoted by index 1 in the schematic diagram.

By changes in the magnitude of (13) in the course of the construction, we can follow the results of the erection of said station 1 . Imagine that after some time, the contractor performing the works $x_{1 i}$ has drawn a share of means intended for his object $P^{\prime}\left(x_{1 i}\right)$. Naturally, before the completion of works at his site, $P^{\prime}\left(x_{1 i}\right)<P\left(x_{1 i}\right)$. At that, the complicacy of his site decreases by the value

$$
P\left(x_{1 i}\right) \ln P\left(x_{1 i}\right)-P^{\prime}\left(x_{1 i}\right) \ln P^{\prime}\left(x_{1 i}\right)=\Delta H\left(x_{1 i}\right)
$$

which is equivalent to

$$
H\left(x_{1 i}\right)-H^{\prime}\left(x_{1 i}\right)=\Delta H\left(x_{1 i}\right)
$$


If he has drawn his means completely, this difference is

$$
\Delta H\left(x_{1 i}\right)=0
$$

In case of a single elementary site, it is clear without additional explanations. However, the suggested approach allows making unambiguous conclusions about the course of the entire project development. In the course of construction, changes taking place are analogous to those considered in each summand of the relation (13). On a certain specific date of the works performance, we can calculate

$$
H^{\prime}\left(x_{11}\right) ; H^{\prime}\left(x_{12}\right) ; H^{\prime}\left(x_{13}\right) ; \cdots ; H^{\prime}\left(x_{1 i}\right) ; \cdots ; H^{\prime}\left(x_{1 m}\right)
$$

A sum of entropies of the drawn funds or performed works is

$$
H^{\prime}\left(x_{1 s}\right)=\sum_{i}^{m} H^{\prime}\left(x_{1 i}\right)
$$

Incompleteness of works at the project equals

$$
\Delta H\left(x_{1 s}\right)=H\left(x_{1 s}\right)-H^{\prime}\left(x_{1 s}\right)
$$

To obtain an habitual estimation in fractions of unity or in percentages, the incompleteness of works can be expressed by a parameter, if we divide both expressions in the right-hand side of (14) by $H\left(x_{1 s}\right)$. Then

$$
F=\frac{H^{\prime}\left(x_{1 s}\right)}{H\left(x_{1 s}\right)}
$$

Repeat once more that the construction incompleteness at all the objects of the first station is determined using the relation (16). We can express this relation in percent, which is habitual for builders, writing a relation

$$
F=\frac{\Delta H\left(x_{1 s}\right)}{H\left(x_{1 s}\right)} \cdot 100 \%
$$

The construction completeness can be expressed by

$$
E=\left[1-\frac{H^{\prime}\left(x_{1 s}\right)}{H\left(x_{1 s}\right)}\right] \cdot 100 \%
$$

It is clear that $E+F$ always equals $100 \%$.

To evaluate the course of construction of all the stations at the same time, we can also obtain a unified estimation using this method. The same method allows obtaining the evaluation of the building of all components of a project of any complicacy.

For this purpose, the expenses for all the stations should be expanded into a matrix

$$
\left|\begin{array}{cccccc}
Q\left(x_{11}\right) & Q\left(x_{21}\right) & \cdots & Q\left(x_{i 1}\right) & \cdots & Q\left(x_{n 1}\right) \\
Q\left(x_{12}\right) & Q\left(x_{22}\right) & \cdots & Q\left(x_{i 2}\right) & \cdots & Q\left(x_{n 2}\right) \\
Q\left(x_{13}\right) & Q\left(x_{23}\right) & \cdots & Q\left(x_{13}\right) & \cdots & Q\left(x_{n 3}\right) \\
\vdots & \vdots & & \vdots & & \vdots \\
Q\left(x_{1 i}\right) & Q\left(x_{2 i}\right) & \cdots & Q\left(x_{i i}\right) & \cdots & Q\left(x_{n i}\right) \\
\vdots & \vdots & & \vdots & & \vdots \\
Q\left(x_{1 m}\right) & Q\left(x_{2 m}\right) & \cdots & Q\left(x_{i m}\right) & \cdots & Q\left(x_{n m}\right)
\end{array}\right|
$$

The sum of the elements of this matrix gives the total cost for the construction of all the stations

$$
\sum_{i}^{n} Q\left(x_{i j}\right)=Q(x)
$$

Dividing all the elements of (19) by (20), we can determine, using the above-described method, the relative 
complicacy of all the stations in the form of total costs and obtain a generalized matrix of static entropies for their characteristics;

$$
\left|\begin{array}{cccccc}
H\left(x_{11}\right) & H\left(x_{21}\right) & \cdots & H\left(x_{i 1}\right) & \cdots & H\left(x_{n 1}\right) \\
H\left(x_{12}\right) & H\left(x_{22}\right) & \cdots & H\left(x_{i 2}\right) & \cdots & H\left(x_{n 2}\right) \\
\vdots & \vdots & & \vdots & & \vdots \\
H\left(x_{1 i}\right) & H\left(x_{2 i}\right) & \cdots & H\left(x_{i i}\right) & \cdots & H\left(x_{n i}\right)
\end{array}\right|
$$

The sum of all entropies for $n$ stations gives the magnitude of the total complicacy of works

$$
\sum_{i}^{n} H\left(x_{i j}\right)=H(x)
$$

As all the works are being accomplished at all the stations at once, their residual complicacies can be determined by performing all computations with this matrix, finding $H^{\prime}(x)$ and determining

$$
E_{x}=\left(1-\frac{H^{\prime}(x)}{H(x)}\right) \cdot 100 \%
$$

In a similar way, we can unambiguously determine the course of accomplishing works with railroad tracks.

The costs for the first section can be written as follows:

$$
Q\left(y_{11}\right)+Q\left(y_{12}\right)+Q\left(y_{13}\right)+\cdots+Q\left(y_{1 s}\right)=Q\left(y_{1}\right)
$$

To obtain a characteristic of the relative complicacy of the performed works, each summand in the left-hand part of (24) must be divided by the expression in the right-hand part. Their sum gives

$$
P\left(y_{11}\right)+P\left(y_{12}\right)+P\left(y_{13}\right)+\cdots+P\left(y_{1 i}\right)+\cdots+P\left(y_{1 s}\right)=1
$$

The entropy of each summand of this sum is

$$
H\left(y_{1 i}\right)=P\left(y_{1 i}\right) \ln P\left(y_{1 i}\right)
$$

and the sum of these entropies is

$$
\sum_{i=1}^{s} H\left(y_{1 i}\right)=H(y)
$$

Their change gives the entropy characterizing the residual complicacy of the works $H^{\prime}\left(y_{1 i}\right)$.

Estimation of the residual complicacy is

$$
F y=\frac{H^{\prime}\left(y_{1 j}\right)}{H\left(y_{1 j}\right)} \cdot 100 \%
$$

The completeness of works is determined by the expression

$$
E=H y-F y=\left[1-\frac{H^{\prime}\left(y_{i j}\right)}{H\left(y_{i j}\right)}\right] \cdot 100 \%
$$

If someone needs a general pattern of the current state of construction of all railroad tracks, it is necessary at first to construct a matrix analogous to (21). Then it is necessary to perform the procedure of computations. In a similar way, an overall estimation of the performance of works for railway stations and railway tracks can be obtained. For that, the specific weight of costs per each group must be taken into account.

$$
Q=Q(x)+Q(y)
$$

Hence,

$$
1=\varphi(x)+\varphi(y)
$$

where

$$
\varphi(x)=\frac{Q(x)}{Q} ; \varphi(y)=\frac{Q(y)}{Q}
$$


Then the total project completion is

$$
E=[1-F x \cdot \varphi(x)-F y \cdot \varphi(y)] \cdot 100 \%
$$

Here we can note the following:

1) In the initial matrices (19) and (21), certain elements can have a zero value.

2) During the performance of works, some elements of the matrices can remain unchanged, if at those sites works were not performed. Similarly, the state of works of the same type at different objects, e.g., assembly, blocking, automation at all the stations, electric lines laying, earthworks, etc., can be estimated separately. To do this, one must perform horizontal summing in matrices of the type of (19) in a necessary line corresponding to a concrete type of works. Then it is needed to compute the fraction of each element by dividing its cost by this sum, and after that to determine the entropy of each element according to the described method (25), find the total complicacy (26) and perform such analysis while the works (27) and (30) are being performed.

The exposed method allows a global and unambiguous estimation of the status of a construction system of any complicacy. For instance, the Ministry of Transport can build, side by side with the railroad, highways, ports, gas stations and many more. The accomplishment of these works at any moment can be unambiguously estimated using the proposed method for the entire Ministry.

We illustrate the application of this method by a specific example. All the computations are presented in a Table 1.

Table 1. Example of evaluation of completeness of complicated construction at an intermediate stage.

\begin{tabular}{|c|c|c|c|c|c|c|c|c|c|}
\hline $\mathrm{N}$ & Name & $\begin{array}{c}\text { Enumeration of } \\
\text { building } \\
\text { objects }\end{array}$ & & & & & & & \\
\hline & & $x_{1}$ & $x_{2}$ & $x_{3}$ & $x_{4}$ & $x_{5}$ & $x_{6}$ & $x_{7}$ & $x_{8}$ \\
\hline 1 & $\begin{array}{c}\text { Financing fraction } \\
\text { for each object } \\
P\left(x_{i}\right)\end{array}$ & 0.05 & 0.125 & 0.175 & 0.25 & 0.08 & 0.12 & 0.13 & 0.07 \\
\hline 2 & $\begin{array}{c}\text { Object complicacy } \\
H\left(x_{i}\right)=P\left(x_{i}\right) \ln P\left(x_{i}\right)\end{array}$ & 0.150 & 0.26 & 0.364 & 0.347 & 0.202 & 0.254 & 0.265 & 0.186 \\
\hline 3 & $\begin{array}{l}\text { Percentage of works } \\
\text { fulfillment at objects, } \\
\qquad k \%\end{array}$ & $28 \%$ & $37 \%$ & $42 \%$ & $63 \%$ & $17 \%$ & $86 \%$ & $91 \%$ & $12 \%$ \\
\hline 4 & $\begin{array}{c}\text { Fraction of fulfilled } \\
\text { works, } \\
P^{\prime}\left(x_{i}\right)=k P\left(x_{i}\right)\end{array}$ & 0.014 & 0.046 & 0.0735 & 0.1575 & 0.036 & 0.1032 & 0.1183 & 0.0084 \\
\hline 5 & $\begin{array}{c}\text { Residual works } \\
\text { at the objects } \\
P\left(x_{0}\right)=P\left(x_{i}\right)-P^{\prime}\left(x_{i}\right)\end{array}$ & 0.036 & 0.079 & 0.1015 & 0.0925 & 0.044 & 0.0168 & 0.0117 & 0.0616 \\
\hline 6 & $\begin{array}{c}\text { Residual complicacy } \\
\text { of works } \\
H\left(x_{0}\right)=P\left(x_{0}\right) \ln P\left(x_{0}\right)\end{array}$ & 0.120 & 0.2 & 0.232 & 0.22 & 0.1374 & 0.0686 & 0.052 & 0.171 \\
\hline 7 & $\begin{array}{l}\text { Relative residual } \\
\text { complicacy } \\
F=\frac{H\left(x_{0}\right)}{H(x)}\end{array}$ & 0.8 & 0.77 & 0.64 & 0.63 & 0.68 & 0.27 & 0.2 & 0.92 \\
\hline 8 & $\begin{array}{c}\text { Efficiency of works } \\
\text { fulfillment at objects } \\
E=\left[1-\frac{H\left(x_{0}\right)}{H(x)}\right] \cdot 100 \%\end{array}$ & $20 \%$ & $23 \%$ & $36 \%$ & $37 \%$ & $32 \%$ & $73 \%$ & $80 \%$ & $8 \%$ \\
\hline
\end{tabular}


The first line of this Table shows the share of financing of each of 8 units. The sum of these shares equals unity. The second line defines relative complicacy of each of the units in compliance with the share of their financing. The third line shows the percentage of executed work at each unit. The fourth line defines the share of executed work on the basis of lines 1 and 3 . The fifth line shows the remaining work at each unit as a difference between lines 1 and 3 . The sixth line defines the complicacy of uncompleted works on the basis of line 5 . The seventh line defines the relative residual complicacy of uncompleted works in shares of unity on the basis of lines 6 and 2. The eighth line shows the efficiency of the executed works expressed percentagewise on the basis of line 7.

By way of example, here we have presented a construction consisting of 8 objects. All the computations in each cell of the Table are explained by formulas placed in the left-hand column.

Determine the sums of the second and sixth lines on the basis of all computations:

$$
\sum_{i}^{8} H\left(x_{i}\right)=2.028 \text { и } \sum_{i}^{8} H_{0}\left(x_{i}\right)=1.201
$$

Hence, the relative residual complicacy of the entire construction is

$$
F=\frac{\sum H_{0}\left(x_{i}\right)}{\sum H\left(x_{i}\right)}=0.59
$$

A unified estimation of the accomplished works is

$$
E=1-F+0.41=41 \%
$$

\section{Conclusion}

A method of unambiguous estimation of the completeness of complicated construction works at any stage of their fulfillment is developed. This creates conditions for simplifying the operative construction management and saving time and means for its realization.

\section{References}

[1] Barsky, E. (2014) Entropy Invariants of Two-Phase Flows. Elselvier, 79-90.

[2] Barsky, E. (2013) Entropy of Two-Phase Flows in the Mode of Separation. Journal of Mining Science, 49, 308-318.

[3] Shannon, C. (1963) A Mathematical Theory of Communication. Moscow, 20-25.

[4] Wilson, A.G. (1987) Entropy in Urban and Regional Modelling. Pion Limited, London. 